\title{
A note on the Definition of $\alpha$-limit Set
}

\author{
Francisco Balibrea ${ }^{1}$, Juan L.G. Guirao ${ }^{2, *}$ and Marek Lampart ${ }^{3}$ \\ ${ }^{1}$ Departamento de Matemáticas. Facultad de Matemáticas. Universidad de Murcia, Región de Murcia, Spain \\ 2 Departamento de Matemática Aplicada y Estadística. Universidad Politécnica de Cartagena, Región de Murcia, Spain \\ ${ }^{3}$ Department of Applied Mathematics \& IT4Innovations. VŠB - Technical University of Ostrava, Czech Republic
}

Received: 23 Jan. 2013, Revised: 24 May. 2013, Accepted: 25 May. 2013

Published online: 1 Sep. 2013

\begin{abstract}
Many phenomena coming from the biology, economy, engineering are modeled using discrete dynamical systems. The concept of backward orbit is an essential concept for understanding the dynamics of the system. In the literature various definitions of the concept of the alpha-limit point (respectively set) have been historically used. The aim of this paper is to analyze the forcing relationships between them via the proof of the valid relationships and the construction of counterexamples for the converse situation in order to clarify the scenario for the computation of these objects. Moreover, we present a discrete dynamical system $(X, f)$ with the following paradoxical behavior: for every point $x \in X$, its alpha-limit set is equal to the whole space $X$; there is a complete negative trajectory of $x$ whose alpha-limit set is equal to a fixed point; there is a complete negative trajectory of $x$ whose alpha-limit set is equal to $X$.
\end{abstract}

Keywords: $\alpha$-limit set, special $\alpha$-limit set, complete negative trajectory.

\section{Introduction and statement of the main results}

A discrete dynamical system (briefly, dynamical system) is defined as an ordered pair $(X, f)$ where $f$ is a continuous map defined on a compact metric space $X$. This kind of systems are the mathematical environment for modelling many phenomena from different areas such as biology, economics and engineering (for further details see [2] and [5] and references therein). To understand the dynamical properties of a system it is necessary to have information on the behavior of the trajectories of any point $x \in X$ under the iteration of $f$. For these purposes many tools are used. In this paper attention is focused on the concept of the $\alpha$-limit set.

The notion of the $\omega$-limit set, i.e. the set of limit points of a trajectory, has been studied in depth in the literature and its definition is not involved at any controversy. The problem with this notion is its topological characterization which is open for the majority of systems (see [9] and references therein). In contrast, the notion of the $\alpha$-limit, which is philosophically its dual notion since it can be regarded as the source of the trajectory of a point, presents some problems from the point of view of its definition due to the many possibilities that can be chosen to construct the backward trajectory and its limits.

Up to now, there have been only a few results concerning $\alpha$-limit sets, mainly in the setting of a system given by continuous maps defined on the unit closed interval and on graphs. X. Jincheng [8] and E. M. Coven, Z. Nitecky [3] found some interesting properties of $\alpha$-limit sets for continuous interval self-maps: More precisely they proved that the set of all $\alpha$-limit points strictly contains the set of non-wandering points; a point $x$ is non-wandering if and only if $x$ belongs to its own $\alpha$-limit set. Finally, H. Cui and Y. Ding, see [6] and [4], studied $\alpha$-limit sets for special types of continuous maps of the interval (expanding Lorentz maps and unimodal maps without homtervals). S. TaiXiang et al [10] proves that special $\alpha$-limits points are contained on the closure of recurrent points. In this setting, the main difficulties come from the fact that the inverse image of a point is generally a set whose cardinality is rather large. Hence, it is quite difficult to follow the backward orbit.

The most general definition of the $\alpha$-limit set (see Definition 1) is very natural, but unfortunately very approximate. This notion was refined firstly by M. W. Hero [7] (see Definition 2) in such a way that only some branches of the backward orbit are considered and

\footnotetext{
*Corresponding author e-mail: juan.garcia@upct.es
} 
recently by F. Balibrea et al [1] (see Definition 4) in such a way that exactly one branch of the backward orbit is considered. The aim of this paper is to state the forcing relationships between these three different notions of the concept of the $\alpha$-limit set by proving valid implications and presenting counterexamples for invalid cases.

The statement of our main results is as follows. The relationship between Definitions 1, 2 and 4 are shown in the scheme below. A missing arrow means that the implication is not valid. Implication (A) is proved by Theorem 1; implication (B) by Theorem 2 and the final one (C) follows from the transitivity. The counterexample for the converse implication of (A) was given by M. W. Hero [7] in Example 1. For the counterexample of the converse to (B) see Theorem 3 and for the final one (C) see Remark 2.

$$
y \in s \alpha_{f}(x) \underset{\nwarrow(\mathrm{B})}{\longrightarrow} \stackrel{(\mathrm{A})}{\longrightarrow}(\mathrm{C}) \nearrow^{y \in \alpha_{f}(x)}
$$

In Section 2 different definitions of the $\alpha$-limit set and some auxiliary results are stated. Section 3 is devoted to the proof of main results.

\section{Definitions of the $\alpha$-limit set and auxiliary results}

The aim of this section is to present three different definitions of the notion of an $\alpha$-limit set, the notation, and some auxiliary results which are needed for stating the proof of the main results and the construction of the counterexamples that are presented in the next section.

The first notion is the classical definition of the $\alpha$-limit set which is analogous to the definition of the $\omega$-limit set.

Definition 1.Let $(X, f)$ be a dynamical system and $x \in X$. A point $y \in X$ is called an $\alpha$-limit point of $x$ under $f$ if and only if there is a strictly increasing sequence of positive integers $\left\{k_{n}\right\}_{n=0}^{\infty}$ and a sequence of points $\left\{y_{n}\right\}_{n=0}^{\infty}$ such that

$$
\begin{aligned}
& \text { 1. } f^{k_{n}}\left(y_{n}\right)=x, \\
& 2 . \lim _{n \rightarrow \infty} y_{n}=y .
\end{aligned}
$$

The set $\alpha_{f}(x)$ of all $\alpha$-limit points of $x$ under $f$ is called the $\alpha$-limit set.

Let us note that the above mentioned definition is equivalent to

$$
\alpha_{f}(x)=\bigcap_{n \geq 0} \overline{\bigcup_{m \geq n} f^{-m}(x)}
$$

and using the same technique the definition of the $\omega$-limit set of $x$ under $f$ (denoted by $\omega_{f}(x)$ ) could be equivalently written as

$$
\omega_{f}(x)=\bigcap_{n \geq 0} \overline{\bigcup_{m \geq n}\left\{f^{m}(x)\right\}} .
$$

M. W. Hero [7] introduced the following concept.

Definition 2.Let $(X, f)$ be a dynamical system and $x \in X$. A point $y \in X$ is called an $s \alpha$-limit point of $x$ under $f$ if and only if there is a sequence of positive integers $\left\{k_{n}\right\}_{n=0}^{\infty}$ and a sequence of points $\left\{y_{n}\right\}_{n=0}^{\infty}$ such that

$$
\begin{aligned}
& \text { 1. } x=y_{0}, \\
& \text { 2. } f^{k_{n}}\left(y_{n}\right)=y_{n-1}, \\
& \text { 3. } \lim _{n \rightarrow \infty} y_{n}=y .
\end{aligned}
$$

The set $s \alpha_{f}(x)$ of all $s \alpha$-limit points of $x$ under $f$ is called the $s \alpha$-limit set.

Many interesting properties were proven by M. W. Hero [7] on $s \alpha$-limit sets for continuous maps on the unit closed interval, see the following proposition. Here, a point $x \in X$ is called almost periodic if for a given open set $U$ containing $x$, one can find an integer $n>0$ such that for any integer $q>0$ there is an integer $r, q \leq r \leq q+n$ with $f^{r}(x) \in U$. A point $x$ is said to be recurrent if it belongs into its $\omega$-limit set.

Proposition 1.Let $([0,1], f)$ be a dynamical system. Then

1.if the sequence $\left\{y_{n}\right\}_{n=0}^{\infty}$ corresponds to Definition 2 then $y \in s \alpha_{f}\left(y_{n}\right)$ for every $n$,

2.if $x \in s \alpha_{f}(y)$ then $f^{n}(x) \in s \alpha_{f}(y)$ for every $n$ and $\omega_{f}(x) \subseteq s \alpha_{f}(y)$,

3.if $x \in X$ is an almost periodic point then $x \in s \alpha_{f}(x)$,

4. if $x \in X$ is a recurrent point then $x \in s \alpha_{f}(x)$.

The following notion, see [2], plays an important role in the final definition of an $\alpha$-limit set.

Definition 3.Let $(X, f)$ be a dynamical system. A complete negative trajectory of a point $x \in X$ is an infinite sequence $\left\{x_{n}\right\}_{n=0}^{\infty}$ such that $x_{0}=x$ and $f\left(x_{n+1}\right)=x_{n}$ for any $n \geq 0$.

The following definition was recently conceived by F. Balibrea et al [1].

Definition 4.Let $(X, f)$ be a dynamical system and $\left\{x_{n}\right\}_{n=0}^{\infty}$ be a complete negative trajectory of a point $x \in X$ for $f$. Then the set $\alpha_{f}\left(\left\{x_{n}\right\}_{n=0}^{\infty}\right)$ of all limit points of $\left\{x_{n}\right\}_{n=0}^{\infty}$ is called the $\alpha$-limit set of $\left\{x_{n}\right\}_{n=0}^{\infty}$.

Now, let $\mathscr{A}$ be any finite set (an alphabet) containing $n$ elements (known as symbols). An infinite word is a map $w: \mathbb{N} \rightarrow \mathscr{A}$, hence it is an infinite sequence $\left(w_{1}, w_{2}, w_{3}, \ldots\right)$ where $w_{i} \in \mathscr{A}$ for any $i \in \mathbb{N}$. The set of all infinite words over the alphabet $\mathscr{A}$ is denoted by $\Sigma_{n}$. 
The set $\mathscr{A}$ is endowed with discrete topology. Then $\Sigma_{n}$ is metrizable by the following metric. For any $x, y \in \Sigma_{n}$ put

$$
d(x, y)= \begin{cases}2^{-k}, & \text { if } x \neq y \\ 0, & \text { otherwise }\end{cases}
$$

where $k$ is the length of the maximal common prefix of $x$ and $y$. Define a shift map $\sigma: \Sigma_{n} \rightarrow \Sigma_{n}$ by

$$
(\sigma(x))_{i}=x_{i+1}
$$

The dynamical system $\left(\Sigma_{n}, \sigma\right)$ is said to be the shift on $n$ symbols.

Now, two elementary results (Lemma 1 and Lemma 2) are formulated, that are very useful and analogous to their $\omega$-limit versions. Note that they are also valid for $s \alpha$-limit set and $\alpha_{f}\left(\left\{x_{n}\right\}_{n=0}^{\infty}\right)$ sets. Recall that set $A \subset X$ is called the invariant if and only if $f(A)=A$.

Lemma 1.Let $(X, f)$ be a dynamical system, $f$ be onto and $x \in X$. Then $\alpha_{f}(x)$ is non-empty, closed and invariant.

Proof. The set $\alpha_{f}(x)$ is non-empty, since the map $f$ is onto. Obviously, in accordance with (1) the set $\alpha_{f}(x)$ is closed.

If $y \in \alpha_{f}(x)$ then in accordance with Definition 1 there is a strictly increasing sequence of positive integers $\left\{k_{n}\right\}_{n=0}^{\infty}$ and a sequence of points $\left\{y_{n}\right\}_{n=0}^{\infty}$ such that $f^{k_{n}}\left(y_{n}\right)=x$ and $\lim _{n \rightarrow \infty} y_{n}=y$. Put $l_{n}=k_{n}-1$ and $z_{n}=f\left(y_{n}\right)$. Then the strictly increasing sequence of positive integers $\left\{l_{n}\right\}_{n=0}^{\infty}$ and the sequence of points $\left\{z_{n}\right\}_{n=0}^{\infty}$ such that $f^{l_{n}}\left(z_{n}\right)=x$ and $\lim _{n \rightarrow \infty} z_{n}=f(y)=z$ are obtained, since

$$
f^{l_{n}}\left(z_{n}\right)=f^{k_{n}-1}\left(f\left(y_{n}\right)\right)=f^{k_{n}}\left(y_{n}\right)=x
$$

and

$$
f(y)=f\left(\lim _{n \rightarrow \infty} y_{n}\right)=\lim _{n \rightarrow \infty} f\left(y_{n}\right)=\lim _{n \rightarrow \infty} z_{n}=z .
$$

Consequently, $\alpha_{f}(x) \subset f\left(\alpha_{f}(x)\right)$. The second inclusion can be proved analogously.

Remark.It must be pointed out that the assumption that $f$ is onto is essential. As an example it is sufficient to put $f(x)=1 / 2$ for any $x \in[0,1]$. Then $\alpha_{f}(1 / 2)=[0,1]$ and $\alpha_{f}(y)=\emptyset$ for any $y \in[0,1 / 2) \cup(1 / 2,1]$, since $f^{-n}(1 / 2)=$ $[0,1]$ and $f^{-n}(y)=\emptyset$ for any $n>0$. Nevertheless, there is only one complete negative trajectory $\{1 / 2\}_{n=0}^{\infty}$ for $f(x)=$ $1 / 2$. So, $\alpha_{f}\left(\{1 / 2\}_{n=0}^{\infty}\right)=\{1 / 2\}$.

One of the most important tools in dynamical systems is conjugacy. That is, two dynamical systems $(X, f)$ and $(Y, g)$ are conjugated if there is a homeomorphism $h: X \rightarrow$ $Y$ such that $g \circ h=h \circ f$. Such homeomorphism is called the conjugacy.
Lemma 2.Let $(X, f)$ and $(Y, g)$ be two conjugated systems with conjugacy $h$. Then $h\left(\alpha_{f}(x)\right)=\alpha_{g}(h(x))$.

Proof. The task is to show that $y \in \alpha_{f}(x)$ if and only if $h(y) \in \alpha_{g}(h(x))$. By Definition $1 y \in \alpha_{f}(x)$ if and only if there is a strictly increasing sequence of positive integers $\left\{k_{n}\right\}_{n=0}^{\infty}$ and a sequence of points $\left\{y_{n}\right\}_{n=0}^{\infty}$ such that $f^{k_{n}}\left(y_{n}\right)=x$ and $\lim _{n \rightarrow \infty} y_{n}=y$. So, there is a strictly increasing sequence of positive integers $\left\{k_{n}\right\}_{n=0}^{\infty}$ and a sequence of points $\left\{h\left(y_{n}\right)\right\}_{n=0}^{\infty}$ such that $g^{k_{n}}\left(h\left(y_{n}\right)\right)=h(x)$ and $\lim _{n \rightarrow \infty} h\left(y_{n}\right)=h(y)$, since $g^{n}=h \circ f^{n} \circ h^{-1}$ for any $n>0$. Hence, $h(y) \in \alpha_{g}(h(x))$. The other inclusion can be proved similarly.

\section{Proof of the main results}

The aim of this section is to state and prove our main results.

\section{Theorem 1.Any s $\alpha$-limit point is $\alpha$-limit.}

Proof.If $y \in s \alpha_{f}(x)$ then in accordance with Definition 2 there is a sequence of positive integers $\left\{l_{n}\right\}_{n=0}^{\infty}$ and a sequence of points $\left\{y_{n}\right\}_{n=0}^{\infty}$ such that $x=y_{0}$, $f^{l_{n}}\left(y_{n}\right)=y_{n-1}$ and $\lim _{n \rightarrow \infty} y_{n}=y$. Put $k_{n}=\sum_{m=1}^{n} l_{m}$. Obviously $\left\{k_{n}\right\}_{n=0}^{\infty}$ is strictly increasing sequence of positive integers and

$$
\begin{gathered}
f^{k_{n}}\left(y_{n}\right)=f^{l_{1}}\left(f^{l_{2}} \ldots\left(f^{l_{n}}\left(y_{n}\right)\right)\right)= \\
f^{l_{1}}\left(f^{l_{2}} \ldots\left(f^{l_{n-1}}\left(y_{n-1}\right)\right)\right)=f^{l_{1}}\left(y_{1}\right)=y_{0}=x
\end{gathered}
$$

so $y \in \alpha_{f}(x)$ ending the proof.

Theorem 2.If $y \in \alpha_{f}\left(\left\{x_{n}\right\}_{n=0}^{\infty}\right)$ then $y \in s \alpha_{f}(x)$.

Proof.If $y \in \alpha_{f}\left(\left\{x_{n}\right\}_{n=0}^{\infty}\right)$ then there is strictly increasing sequence $\left\{l_{n}\right\}_{n=0}^{\infty}$ of positive integers such that $x_{l_{n}} \rightarrow y$ for $n \rightarrow \infty$. Put $k_{n}=l_{n+1}-l_{n}$ and $y_{n}=x_{l_{n+1}}$ for each $n$. Hence, $x=x_{0}, f^{k_{n}}\left(y_{n}\right)=y_{n-1}$ and $\lim _{n \rightarrow \infty} y_{n}=y$. Consequently, $y \in s \alpha_{f}(x)$.

Theorem 3.There is a dynamical system $(X, f)$ such that for any $x \in X$

1. $\alpha_{f}(x)=s \alpha_{f}(x)=X$,

2.there is a complete negative trajectory $\left\{x_{n}\right\}_{n=0}^{\infty}$ and $\alpha_{f}\left(\left\{x_{n}\right\}_{n=0}^{\infty}\right)=\{p\}$ where $p$ is the fixed point of $f$,

3.there is a complete negative trajectory $\left\{x_{n}\right\}_{n=0}^{\infty}$ and $\alpha_{f}\left(\left\{x_{n}\right\}_{n=0}^{\infty}\right)=X$.

Proof.Let $(X, f)=\left(\Sigma_{2}, \sigma\right)$ and $y, x$ be arbitrary points in $\Sigma_{2}$. Denote by $[x]_{n}$ the block $x_{1} x_{2} \ldots x_{n}$ of length $n$.

1.Let $y_{n}=[y]_{n}[y]_{n-1}[y]_{n-2} \ldots[y]_{1} x$ be a sequence of elements in $\Sigma_{2}$. Let $\left\{k_{n}\right\}_{n=0}^{\infty}$ be a sequence of positive integers such that $k_{n}=n$. Hence, $y_{0}=x$, $\sigma^{k_{n}}\left(y_{n}\right)=y_{n-1}$ and $\lim _{n \rightarrow \infty} y_{n}=y$. Since the point $y$ was arbitrarily chosen it may be now deduced $s \alpha_{\sigma}(x)=\Sigma_{2}$. The second equality follows from Theorem 1 . 
2.It is sufficient to take a complete negative trajectory $\left\{x_{n}\right\}_{n=0}^{\infty}=\left\{[0]_{n} x\right\}_{n=0}^{\infty}$. Obviously, this trajectory tends to the fixed point $000 \cdots=\overline{0}$ in $\Sigma_{2}$.

3.Let $e \in \Sigma_{2}$ be a point with a dense orbit, constructed as a concatenation of all blocks of all lengths (for more details see [5]). Then put $\left\{x_{n}\right\}_{n=0}^{\infty}=\left\{[e]_{n} x\right\}_{n=0}^{\infty}$. Consequently, $\alpha_{\sigma}\left(\left\{x_{n}\right\}_{n=0}^{\infty}\right)=\Sigma_{2}$.

Example 1.Here the $\alpha$-limit sets for the tent map $T(x)=1-|1-2 x|$ defined on $[0,1]$ are discussed. Firstly, the tent map is onto, so in accordance with Lemma 1 $\alpha_{T}(x)$ it is non-empty, closed and invariant. It is possible to find an inverse image of any point in any non-degenerate interval for $T$, since $T$ is transitive. So, for any $x \in[0,1], \alpha_{T}(x)=[0,1]$ is obtained. The tent map restricted to the Cantor ternary set is conjugated to $\left(\Sigma_{2}, \sigma\right)$ (see [5]). Hence, in accordance with Lemma 2 and Theorem $3 \alpha$-limit sets are more complex with respect to the complete negative trajectory. So, it is possible that they are finite or uncountable.

\section{Acknowledgements}

The first two authors were partially supported by MCYT/FEDER (No. MTM2011-22587).

The third author was supported by the European Regional Development Fund in the IT4Innovations Centre of Excellence project (CZ.1.05/1.1.00/02.0070), by the Grant Agency of the Czech Republic (No. P201/10/0887) and the Ministry of Education of the Czech Republic (No. MSM6198910027).

\section{References}

[1] F. Balibrea, G. Dvorníková, M. Lampart and P. OPROCHA, On negative limit sets for one-dimensional dynamics, Nonlinear Analysis Series A: Theory, Methods \& Applications, 75, 3262-3267 (2012).

[2] L. S. Block AND W. A. Coppel, Dynamics in one dimension, Lecture notes in Mathematics, Springer-Verlag, 1513, (1992).

[3] E. M. Coven And Z. NiteCKI, Non-wandering sets of powers of maps, Ergod. Th. \& Dyn. Sys, 1, 9-31 (1981).

[4] H. CUI AND Y. Ding, The $\alpha$-limit sets of a unimodal map without homtervals, Topology Appl., 157, 22-28 (2010).

[5] R. L. Devaney, An introduction to chaotic dynamical systems, Addison-Wesley Studies in Nonlinearity, AddisonWesley Publishing Company Advanced Book Program, Redwood City, CA, (2003).

[6] Y. Ding, Renormalization and $\alpha$-limit set of expanding Lorenz map, Discrete Contin. Dyn. Sys., 29, 979-999 (2011).

[7] M. W. HeRo, Special $\alpha$ limit points for maps of the interval, Proc. Amer. Math. Soc., 116, 1015-1022 (1992).

[8] X. JINCHENG, The attracting centre of a continuous self-map of the interval, Ergod. Th. \& Dyn. Sys, 8, 205-213 (1988).

[9] V. ŠPITALSKÝ, Omega-limit sets in hereditarily locally connected continua,Topology Appl., 115, 1237-1255 (2008).
[10] S. TaiXiang, X. HongJian and L. Hailan, Special $\alpha$-limit points and unilateral $\gamma$-limit points for graph maps, Science China Math., 54, 2013-2018 (2011).

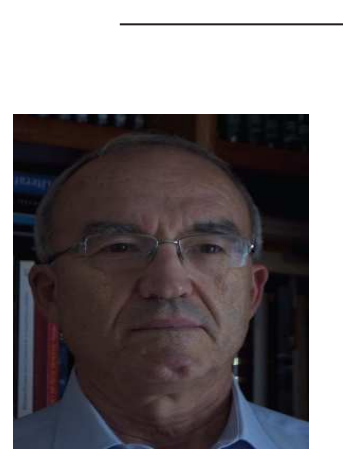

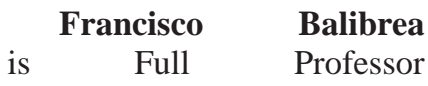
of Mathematical Analysis at University of Murcia in Spain. He founded more than 30 year ago the Dynamical System Group of the Region of Murcia. He has supervised more than 15 PhD's and writes more than 100 paper publish in the best research journal covering a wide range of topics of topological low dimensional dynamics and difference equations.

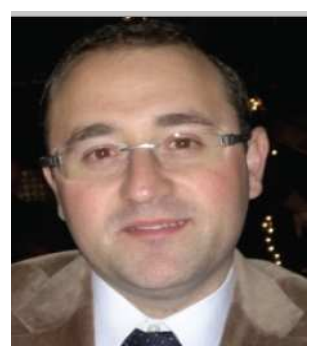

Juan Luis García Guirao is Full Professor of Applied Mathematics at Universidad Politécnica de Cartagena in Spain. In 2010, he became in the youngest Mathematics Full Professor in Spain with 33 years old. Author of more than 60 research papers published in the best journal he has supervised 4 PhD's and more than 10 Master Thesis. He belongs to the Editorial Board of several journals, between them MATCH Commun. Math. Comput. Chem. ranked in first position of the JCR 2010 list (Interdisciplinary Mathematics).

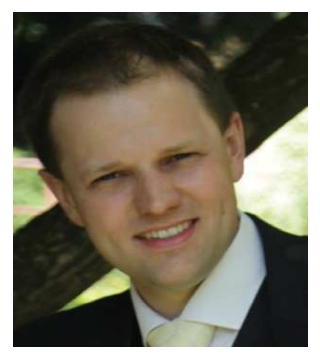

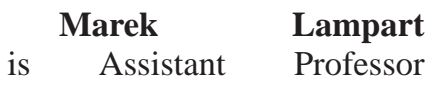
at Department of Applied Mathematics and Junior Researcher at IT4Innovations VŠB-Technical University of Ostrava, Czech Republic. He received the $\mathrm{PhD}$ degree in "Pure Mathematics" at the Silesian University of Opava (Czech Republic). $\mathrm{He}$ is referee of several international journals in the frame of pure and applied mathematics. His main research interests are: dynamical systems on compact metric spaces, applied dynamical systems in economy and mechanics. 